
\title{
25 Research Soure \\ Full-Endoscopic Lumbar Discectomy for Two Contiguous Level Adolescent Lumbar Disc Herniation
}

lu mao ( $\sim$ bobai0008@163.com )

Southeast University Zhongda Hospital

Bin Zhu

Peking University

Xiaotao Wu

Southeast University

Feng Wang

Southeast University

Cong Zhang

Southeast University

Jie Zhao

Shanghai Jiao Tong University School of Medicine

Research article

Keywords: Adolescent, Lumbar, Disc herniation, Endoscopic, Surgery

Posted Date: June 29th, 2020

DOI: https://doi.org/10.21203/rs.3.rs-34230/v1

License: () (1) This work is licensed under a Creative Commons Attribution 4.0 International License. Read Full License 


\section{Abstract}

Background: Symptomatic lumbar disc herniation is rarely seen among adolescents. Adolescent lumbar disc herniation (ALDH) accounts for $0.5 \%$ to $6.8 \%$ of all those treated. Evidently, to our knowledge, no studies have emphasized the operative technique to treat two contiguous level adolescent lumbar disc herniation simultaneously. In this study, we aim to investigate the feasibility and advantages of one-stage full-endoscopic lumbar discectomy $\mathbb{F E L D \otimes f o r ~ t w o ~ c o n t i g u o u s ~ l e v e l ~ A L D H . ~ T h i s ~ i s ~ t h e ~ f i r s t ~ p a p e r , ~ t o ~ m y ~}$ knowledge, dealing with two contiguous level ALDH simultaneously with one-stage full-endoscopic lumbar discectomy.

Methods: Between January 2014 and December 2019, patients received FELD surgery for lumbar disc herniation (LDH) in 2 main minimally invasive spine center of China were selected for screening of this study. Data of 5877 cases were retrospectively analyzed (2780 cases and 3097 cases, respectively). The inclusion criteria were patient under 21-year old, two contiguous level symptomatic lumbar disc herniation received one-stage 2 level PELD surgery. Visual analog scale (VAS) scores and modified Macnab criteria were used to assess the preoperative and postoperative clinical results.

Results: 11 patients were enrolled in this study $(0.19 \%, 11 / 5877), 8$ patients were male and 3 were female. 9 patients with LDH in the same side underwent single-incision 2 level FELD surgery via transforaminal approach. 2 patients with LDH in different side underwent FELD surgery via combined transforaminal and interlaminar approach. There were no immediate perioperative complications. The visual analog scale (VAS) decreased significantly in both early and late follow-up evaluations and these score demonstrated significant improvement in late follow-up $(P<0.01)$. For the modified MacNab criteria, the final outcome results were excellent in 9 patients (81.8\%), good in 1 patients $(0.9 \%)$, fair in 1 patients $(0.9 \%)$, and the overall success rate was $90.9 \%$.

Conclusion: One-stage full-endoscopic lumbar discectomy for 2 level symptomatic ALDH is an effective and less invasive method, which can be considered as an alternative good technique for selected adolescent patients.

\section{Background}

Symptomatic lumbar disc herniation is rarely seen among adolescents. Adolescent lumbar disc herniation (ALDH) accounts for $0.5 \%$ to $6.8 \%$ of all those treated[1-4]. Evidently, two contiguous level adolescent lumbar disc herniation is less common. So far, the treatments available for ALDH and the effect of each treatment have not been fully reviewed [2].Patients who have failed in nonsurgical therapy, suffering from the progressive neurologic deficit or debilitating pain, surgical treatment is usually recommended. Nowadays, even though full-endoscopic lumbar discectomy (FELD) has rapidly evolved and gained popularity, but the efficacy of FELD is still controversial for symptomatic two contiguous level $\operatorname{ALDH}[5,6,10]$. To our knowledge, currently, no studies have emphasized the operative technique to treat two contiguous level ALDH simultaneously. In this retrospective study, we aim to investigate the 
feasibility and advantages of one-stage full-endoscopic lumbar discectomy for two contiguous level ALDH.

\section{Methods}

\section{Patients}

This study was approved by the Ethic Committee of The Zhongda Hospital Southeast University(CODE: 2020ZDSYLL080-P01). The minimally invasive spine department of these 2 hospitals are main minimally invasive spine centers of China (more than 800 FELD surgeries per year) .

Between January 2014 and December 2019, a total of 5877 patients underwent FELD for symptomatic lumbar disc herniation, 2780 cases in Zhongda Hospital Southeast University and 3097 cases in Peking University Third Hospital. Data of these patients were retrospectively analyzed. The inclusion criteria of this study were: (1) patient under 21-year old; (2) radicular leg pain caused by multilevel lumbar disc herniation; (3) failure of conservative measures for a minimum of three months; (4) progressive neurologic deficit or debilitating pain.

The exclusion criteria were: (1)the presence of segmental instability; (2) cauda equina syndrome; (3) patients with other serious systemic diseases. (4) patients with incomplete data or patients who were lost to follow-up.

Surgical Procedure

All surgeries were performed by the experienced surgeon (660 per year). Transforaminal approach ("TESSYS" techniques) described by Hoogland et al.[7] and interlaminar approach(YESS"techniques) described by Ruetten et al.[8] were applied. All the surgeries were performed under local anaesthesia ( $0.5 \%$ lidocaine)and sedated with midazolam and fentany, which allow for monitoring of any changes of the patients' symptoms and make it possible for surgeons to get instant feedback from patients so as not to cause damage to any neural structures. The patient was placed in a prone position or lateral position on the radiolucent operating table. To determine an appropriate entry point and approach angle, preoperative images should be used to calculate and determine an appropriate puncture point and approach angle. (Fig. 1A). The point also depends on the location of the herniated disc, patient's body size and land foraminal dimension.

\section{Single-incision 2-level transforaminal FELD:}

For patient with herniated disc in the same side (all levels)(Fig.1 A,B), transforaminal FELD via single incision was applied. The single puncture point is determined by the intersection of two line, which the needle tip directed downwards making an angle of $10^{\circ}-30^{\circ}$ with the upper endplate of the distal vertebrate. Under lateral x-ray fluoroscopy(Fig.1C), the line connecting the superior facet margin was marked as the safety line[7-8]. After routine disinfection, the single puncture point was generally 10-14 $\mathrm{cm}$ from the midline, and a long 18-gauge spinal needle was inserted from the entry point toward the 
midline under intermittent fluoroscopic guidance. The superior facet joint was used as anatomic marker to avoid puncturing injuries and compressions to the existing nerve root. The needle was then replaced with a 1-mm-diameter guide wire. After a 10-mm skin incision was made close to the guide wire, and serial dilators and an obturator are introduced. The foramina were enlarged with a saw (Fig. 1C,D,E,F) . The surgeons usually operate the lower level at first. The working cannula is the beveled type with an 8 mm outer diameter.

Since the intervertebral foramen was adequately enlarged with a saw, additional maneuvers like levering the working cannula to make it more horizontal, downward or upward tilting could be easily achieved. The intervertebral disc was excised with the aid of an endoscope. An endoscopic rongeur was used to remove the degenerated nucleus, which was then sent for pathological examination[7-9]. The decompression was concluded when the dura and spinal nerves were clearly visibly decompressed and the pulsation of the dural tube and the nerve root was confirmed, which was an important indicator of thorough decompression and surgical termination[9-10]. After complete removal of disc, the ventral dura demonstrated free pulsation with the Valsalva maneuver. Annuloplasty was typically performed at the end of the discectomy and minimal damage to the posterior structures. Hemostasis was performed with bipolar diathermy. The endoscope was removed after no active bleeding was confirmed[9].

\section{Combined transforaminal and interlaminar FELD:}

For patients with L4-L5 disc herniation in one side and L5-S1 disc herniation in the other side, one-stage transforaminal approach for L4-L5 level combined interlaminar approach for L5-S1 level was applied (Fig.2 A-F). [8].

\section{Postoperative Management}

After resting in bed for 1 days postoperatively, the patients could have off-bed activity appropriate with a protective belt and begin lumbodorsal muscle exercise and straight leg-raising exercise. One week later, the patients resumed light physical labor. In the rehabilitation period, to achieve favorable healing of the ruptured annular fibrosis and decrease recurrence of disc herniation, wearing the lumbar back brace approximately 2-4 week was recommended.

\section{Clinical assessment}

Clinical chart review and telephone survey was performed. The outcomes of symptoms were analyzed using the VAS scores for low back and leg pain pre-operatively and immediately, at three and annually thereafter by an independent assessor. It is a continuous scale composed anchored by a score of zero, indicated no pain, and a score of 10 , represented the worst pain. Modified Macnab criteria for the patient satisfaction[11].Excellent indicates no pain and no restriction of movement, allowing the patient to work normally; good indicates occasional pain, allowing the patient to work normally; fair indicates slight progress; poor indicates no progression.

Statistical analysis 
The pre-operative and postoperative clinical results were statistically analysed using the Wilcoxon signedrank test to compare scores.

\section{Results}

\section{Demographic Data}

In this retrospective study, a total of 11 patients (female/male: 3/9) were consecutively enrolled with a follow-up period of 17.3 months in average. $0.19 \%$ of all FELD surgeries in these 2 minimally invasive spine center. 4 cases affected the L3- L4 /L4- L5 level, and 7 cases affected the L4- L5 /L5-S1 level. The average duration of symptoms was 17.7 months.

\section{Clinical Outcomes}

All operations were successfully performed. The mean operation time was $68.4 \pm 9.5$ minutes. The mean length of hospital stay was $4.5 \pm 0.7$ days. No serious complications occurred. Clinical outcomes in follow-up were measured for all patients according to the criteria used by the VAS and modified Macnab criteria. The Low back pain and leg pain were significantly relieved immediately after surgery in all patients. The significantly improved VAS occurred between pre-operative and early follow-up assessments with little changes between early and late follow-up $(P<0.01)$. The relief of pain during walking, standing, and sitting positions were identified. MRI was performed when the patient complained of newly developed radiating pain. For all other patients without new symptoms, only a clinical follow-up was conducted.

\section{Discussion}

Disease occurring under 21-years of age is termed 'adolescent'[12-14]. But at what age there is a relevant distinction from adult disease is unclear. Great care should be taken when operating on the immature spine due to that it is unknown whether operating on the immature spine may increase their risk for having spinal surgery in the future[13]. Additionally, delaying surgery for conservative treatment is warranted, but for how long remains unclear.

Appropriate conservative treatment including (bed rest, physical therapy and (NSAIDs) is the first choice for adolescents, but the young patients do not respond as well to nonsurgical treatment as adults due to adolescent disk material often remains well hydrated[13-15]. The surgical aim of treatment for ALDH is achieving of appreciable pain relief and function improvement. Mixter and Barr [16], published the first report of a herniated nucleus pulposus in a child in 1934, with another report of surgical treatment for a 12-year-old boy by Wahren in 1945 [17]. Traditionally, open discectomy (OD) and microendoscopy discectomy (MED) was employed as the standard operation for ALDH [18,19].

In recent years, minimally invasive techniques are an attractive alternative to open discectomy (OD) and microendoscopy discectomy (MED) with a view to improving management of ALDH patients[18,19]. 
Endoscopic techniques have been widely used for ALDH since the first introduction by Ruetten et al [8]. It has unique advantages of minimizing trauma to the normal spinal structures, reducing intraoperative bleeding and allowing earlier return to work. Most studies[10,12,13] have since been published on the surgical management of single level (especially L4-L5 or L5-S1) disk herniation in children and adolescents. Due to the sample size of 2 contiguous level ALDH is relatively small and rarity of its incidence, even though FELD has rapidly evolved and gained popularity, but, the efficacy of FELD is still debatable for contiguous ALDH. To our knowledge, currently, those studies had not mentioned one-stage operation of full-endoscopic lumbar discectomy for 2 contiguous level ALDH simultaneously[9-14].

Anatomically, inclination of L5-S1 disc spaces steeper than the L4-L5, which making single entry puncture point is enough to perform PELD at both the L4-L5 and L5-S1 levels. According to described technique[5,10], in cases of L4/L5- L5/S1 ALDH, under fluoroscopic guidance the meeting point of two lines crossing the $L 5$ and $S 1$ facet joint, indicating the point through which the surgeon can perform PELD for two level ALDH. In case of L3/L4-L4/L5 herniation, a small single skin puncture point is also possible, which rely on the technique of rod adjustment of a working cannula and targeted fragmentectomy. The favorable indications for one-stage operation of transforaminal full-endoscopic lumbar discectomy are same-side two level lumbar disc herniations causing unilateral radicular leg pain[10]. However, the transforaminal approach at L5-S1 has limitations in cases of high iliac crests, small intervertebral foramen, large migrated disc herniations and different side lumbar disc herniations[12]. In this study, 2 patients underwent one level transforaminal endoscopic lumbar discectomy combine one level endoscopic interlaminar discectomy. Because of this 2 pantients indicated different side lumbar disc herniations causing bilateral radicular leg pain.

ALDH is a rare disease with an incidence of only $1 \%-5 \%$. Approximately $93 \%$ of symptomatic disk herniations occur predominantly at vertebral levels L4/L5 and L5/S1, other levels and 2 contiguous level disease recognized, but uncommon. Wang et al. [20] revealed that among 121 adolescents patients, L4/5 disease accounted for $50.4 \%(n=61)$ of patients, L5/S1 for $34.7 \%(42 / 61)$, L3/4 for $3.3 \%(4 / 61), L 4 / 5+$ L5/S1 for $10.7 \%$ (13/61), and L3/4 + L4/5 for $0.8 \%$. However, this cases have undertook the single level traditional open discectomy such as open discectomy (OD) and microendoscopy discectomy (MED).

The distinguishing feature of adult LDH was a result of age-related degenerative process of the spine. However, ALDH must be explained another cause such as micro-trauma, because degeneration is infrequent in adolescents[22-24]. But in actual fact the pathogenesis of ALDH is unclear, trauma or sports-related incidents, genetics and dysfunctional bio-mechanical conditions ( being overweight, or being tall, congenital lumbosacral malformations ) are likely contributory [21-25]. This study indicated that students accounted for $77.7 \%$, we speculate that hours spent sitting is a major risk factor due to increased axial load. In our study, flattening of the sagittal lumbar curvature happened in 7 cases (63.6\%). Other studies demonstrated that flattened spines are often associated with degeneration of multiple discs and back muscle weakness, which further significantly decreases spinal flexibility and stability[25,26,27] . 
Several points should be kept in mind. (1) placement and rod adjustment of working cannula precisely, (2) toward the target compressing element of the disc directly, (3) require proper training and suitable patient selection.

Despite the sample size is relatively small because of rarity of its incidence with retrospective design, the absence of a control group and the follow-up period is too short to comment on the subsequent degeneration of the disc, our study suggests that one-stage operation of full-endoscopic lumbar discectomy for 2 contiguous level ALDH is an effective and less invasive method. To overcome the limitations, further studies are wanted to determine long-term therapeutic effect of one-stage operation of full-endoscopic lumbar discectomy for 2 contiguous level ALDH.

\section{Conclusion}

This study's data suggest that one-stage operation of full-endoscopic lumbar discectomy for 2 contiguous level ALDH is an effective and less invasive method that avoids open surgery and provides another stable and acceptable treatment option in proper patient selection.

\section{Abbreviations}

ALDH囚Adolescent lumbar disc herniation

FELDQFull-endoscopic lumbar discectomy

OD: Open discectomy

MED: Microendoscopy discectomy

VAS: Visual analog scale

\section{Declarations}

\section{Ethics approval and consent to participate}

We obtained ethics approval from the Research Ethics Committee of Zhongda Hospital Southeast University(CODE: 2020ZDSYLL080-P01). All procedures performed in studies involving human participants were in accordance with the ethical standards of the institutional and/or national research committee. For this retrospective study, formal consent is not required.

\section{Consent for publication}

Not applicable.

\section{Availability of data and materials}


The datasets used and/or analyzed during the current study are available from the corresponding author on reasonable request.

\section{Competing interests}

The authors declare that they have no competing interests.

\section{Authors' contributions}

LM: Drafting the manuscript and surgical treatment. BZh:Drafting the manuscript and surgical treatment XTW: Surgical treatment and conception, design and manuscript revision. FW: Data analysis and drafting the manuscript. CZ: Acquisition of the data. JZ: Analysis and interpretation of the data. All authors agree to the final submitted manuscript and declare that they have no conflict of financial interest.

\section{Acknowledgements}

We are grateful to Dr. Yun-Tao Wang, Dr.Su-Yang Zhuang, Dr. Xin Hong, Dr. Zan-Li Jiang, Dr. Shao-Dong Zhang, Dr. Xin-hui Xie, Dr. Lei Zhu, Dr. Xiaohu Wang, Dr.Kun Wang, Dr. Pei Zhang and Dr. Kaixi Liu for their contributions to this manuscript.

\section{We have draw "figure 1D-F, 2D-E" by ourselves, so we own the copyright.}

\section{Authors' information}

1.Department of Spine Surgery, Zhongda Hospital, School of Medicine, Southeast University, Nanjing, Jiangsu Province, China.

2.Pain medicine center, Peking University Third Hospital, Beijing, China.

3.Shanghai Key Laboratory of Orthopaedic Implants, Department of Orthopaedic Surgery, Ninth People's Hospital, Shanghai Jiaotong

University School of Medicine, 639 Zhizaoju Rd, Shanghai,China.

\section{References}

1. F. Lavelle, A. Bianco, R. Mason, R.R. Betz, S.A. Albanese (2011) Pediatric disk herniation, Am. Acad. Orthop. Surg. 19:649-656, https://doi.org/10.5435/ 00124635-201111000-00001

2. Samartzis D, Karppinen J, Mok F, Fong DY, Luk KD, Cheung KM (2011) A population-based study of juvenile disc degeneration and its association with overweight and obesity, low back pain, and diminished functional status. J Bone Joint Surg Am 93(7):662-670

3. Peng B, Hou S, Wu W, Zhang C, Yang Y (2006) The pathogenesis and clinical signifcance of a highintensity zone (HIZ) of lumbar intervertebral disc on MR imaging in the patient with discogenic low back pain. Eur Spine J 15(5):583-587 
4. Tian-Hang Xie, Jian-Cheng Zeng , Zhu-Hai Li , Liang Wang , Hong-Fei Nie , Hu-Shan Jiang , Yue-Ming Song , Qing-Quan Kong. (2017) Complications of Lumbar disc herniation following full-endoscopic interlaminar lumbar discectomy: A Large, Single-Center, Retrospective Study. Pain Physician ; 20:E379-E387.

5. Lee, S-K Kim, S-H Lee, W-J Kim (2007) Percutaneous endoscopic lumbar discectomy for migrated disc herniation: classification of disc migration and surgical approaches.Eur Spine J 16:431-437

6. Kambin P , Gellman H (1983) Percutaneous Lateral Discectomy of the Lumbar Spine A Preliminary Report [J]. Clinical Orthopaedics and Related Research, 1983, (174):127-132.

7. Hoogland T, Schubert M, Miklitz B, Agnes R( 2006囚Transforaminal posterolateral endoscopic discectomy with or without the combination of a low-dose chymopapain: A prospective randomized study in 280 consecutive cases. Spine (Phila Pa 1976) ; 31(24):E890-E897.

8. Ruetten S, Komp M. A new full-endoscopic technique for the interlaminar operation of lumbar disc herniations using 6-mm endoscopes: Prospective 2-year results of 331 patients $\$ 2006 \rrbracket$ Minim Invasive Neurosurg ; 49:80-87

9. Wang K, Hong X, Zhou BY, Bao JP et al (2015) Evaluation of transforaminal endoscopic lumbar discectomy in the treatment of lumbar disc herniation. Int Orthop 39(8):1599-1604

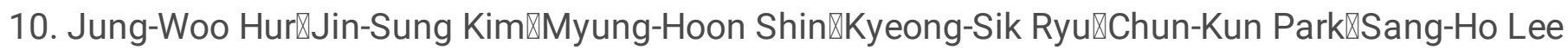
\2014『Percutaneous endoscopic lumbar discectomy and annuloplasty for lumbar disc herniation at the low two contiguous levels: single-portal, double surgeries. J Neurol Surg A;75:381-385

11. Macnab I (1971) Negative disc exploration An analysis of the causes of nerve-root involvement in sixty-eight patients. J Bone Joint Surg Am 53:891-903

12. Tu, Z. , Wang, B. , Li, L. , Li, Y. , Li, T. (2018). Early experience of full-endoscopic interlaminar discectomy for adolescent lumbar disc herniation with sciatic scoliosis. Pain Physician, 21(1), E63E70.

13. Xu, Z. , Liu, Y. , Chen, J. (2017). Percutaneous endoscopic interlaminar discectomy for L5/S1 adolescent lumbar disc herniation. Turkish neurosurgery, 28(6)::923-28.

14. Li, C. Jiang, X. Mu, W. Lan, Y. Zhou, C. Li (2018)Comparison of MED and PELD in the treatment of adolescent lumbar disc herniation: a 5-Year retrospective follow-up, World Neurosurg. 112 e255e260.

15. Lee DY, Ahn Y, Lee SH. (2006). Percutaneous endoscopic lumbar discectomy for adolescent lumbar disc herniation: surgical outcomes in 46 consecutive patients. Mount Sinai Journal of Medicine New York, 73(6), 864.

16. Mixter WJ, Barr JS(1934) Rupture of the intervertebral disc with involvement of the spinal canal. N Engl J Med :211:210-215

17. Wahren $\mathrm{H}(1945)$ Herniated nucleus pulposus in a child of twelve years. Acta Orthop Scand :16:40-42

18. Lagerbäck, P. Elkan, H. Möller, A. Grauers, E. Diarbakerli, P. Gerdhem (2015) An observational study on the outcome after surgery for lumbar disc herniation in adolescents compared with adults based on the Swedish Spine Register, Spine J. 15:1241-1247, https://doi.org/10.1016/j.spinee.2015.02.024 
19. Wu X, Zhuang S, Mao Z, Chen H (2006) Microendoscopic discectomy for lumbar disc herniation: surgical technique and outcome in 873 consecutive cases. Spine 31(23):2689-2694

20. Hongwei Wang, Jiwei Cheng, Hong Xiao, Changqing Li, Yue Zhou (2013) Adolescent lumbar disc herniation: Experience from a large minimally invasive treatment centre for lumbar degenerative disease in Chongqing, China. Clinical Neurology and Neurosurgery 115: 1415-1419

21. Fakouri, C. Nnadi, B. Boszczyk, A. Kunsky, F. Cacciola (2009) When is the appropriate time for surgical intervention of the herniated lumbar disc in the adolescent? J. Clin. Neurosci. $16: 1153-$ 1156, https://doi.org/10.1016/j.jocn.2009.01.023.

22. D. Montejo, J.Q. Camara-Quintana, D. Duran, J.M. Rockefeller, S.B. Conine, A.M. Blaise, K.T. Kahle, M.L. DiLuna (2018) Tubular approach to minimally invasive microdiscectomy for pediatric lumbar disc herniation, J. Neurosurg. Pediatr. 21:449-455, https://doi.org/10.3171/2017.11.PEDS17293.

23. Korovessis $P$, Stamatakis $M$, Baikousis $A(1999)$ Segmental roentgenographic analysis of vertebral inclination on sagittal plane in asymptomatic versus chronic low back pain patients. Journal of Spinal Disorders12(2):131-137

24. Endo K, Suzuki H, Tanaka H, Kang Y, Yamamoto K(2010) Sagittal spinal alignment in patients with lumbar disc herniation. European Spine Journal 19(3):435-438

25. Dang L, Liu Z $₫ 2010 \rrbracket A$ review of current treatment for lumbar disc herniation in children and adolescents. European Spine Journal 19(2):205-214

26. Zhang YG, Sun Z, Zhang Z, Liu J, Guo X (2009) Risk factors for lumbar intervertebral disc herniation in Chinese population: a case-control study. Spine (Phila Pa 1976)34(25):E918-922

27. Lu Mao, Jie Zhao, Ke-rong Dai, Li Hua , Xiao-jiang Sun(2013) Bilateral decompression using a unilateral pedicle construct for lumbar stenosis. Int Orthopedics 38(3):573-578

\section{Figures}




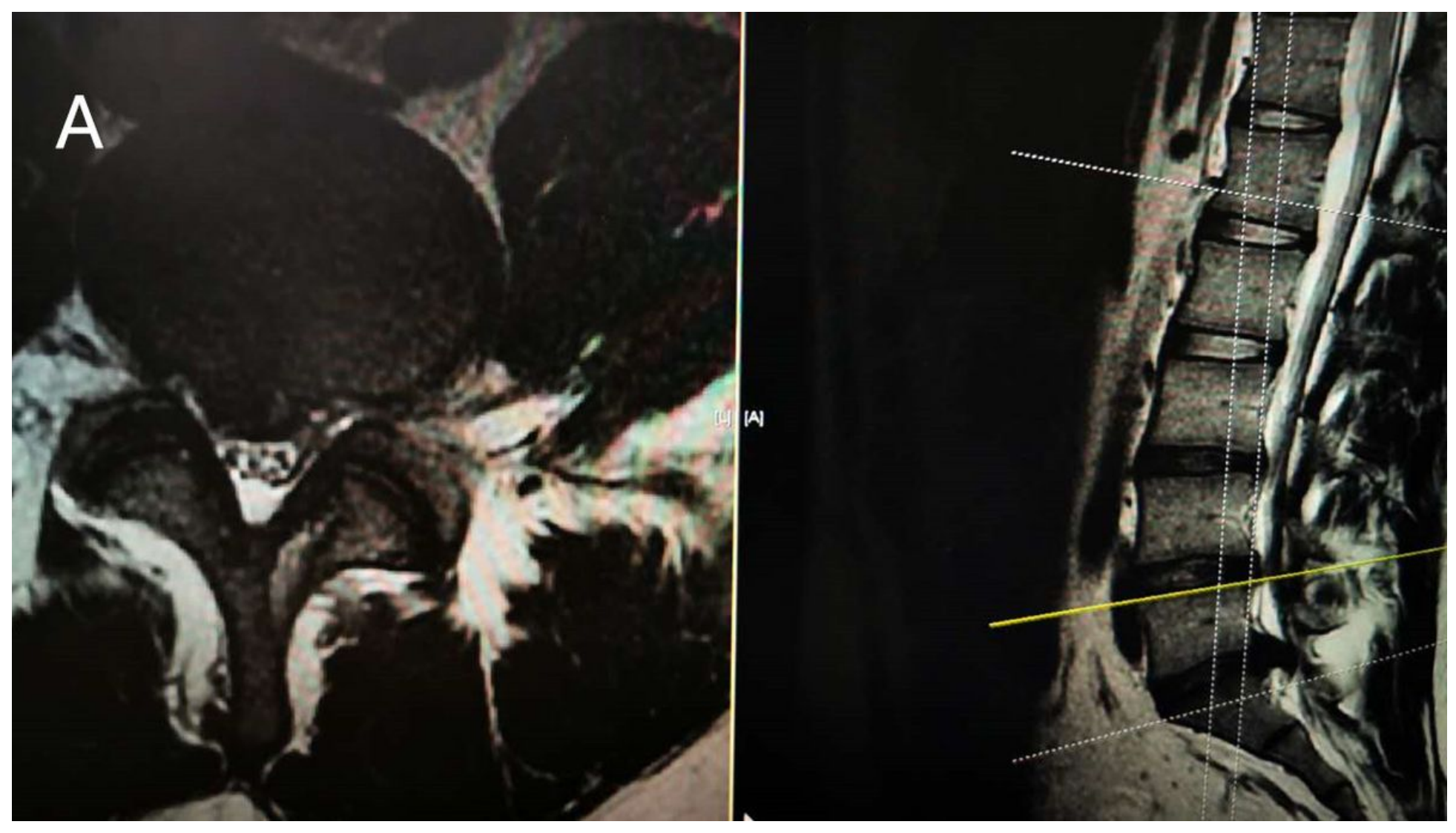

Figure 1

MRI of a 17-year-old woman with two-lever lumbar disc herniation (L3/L4)

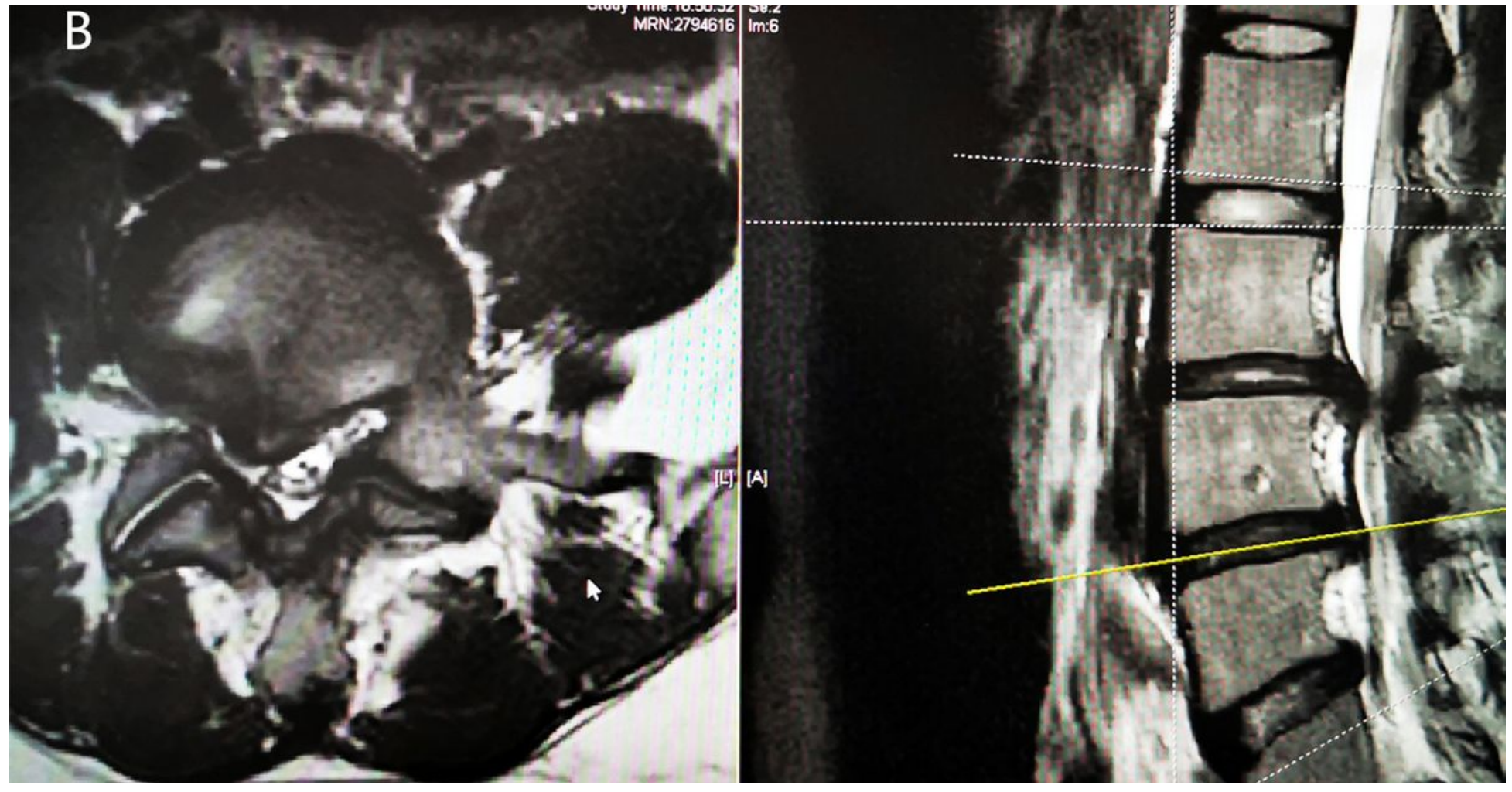

Figure 2 
MRI of a 17-year-old woman with two-lever lumbar disc herniation ( L4/L5)

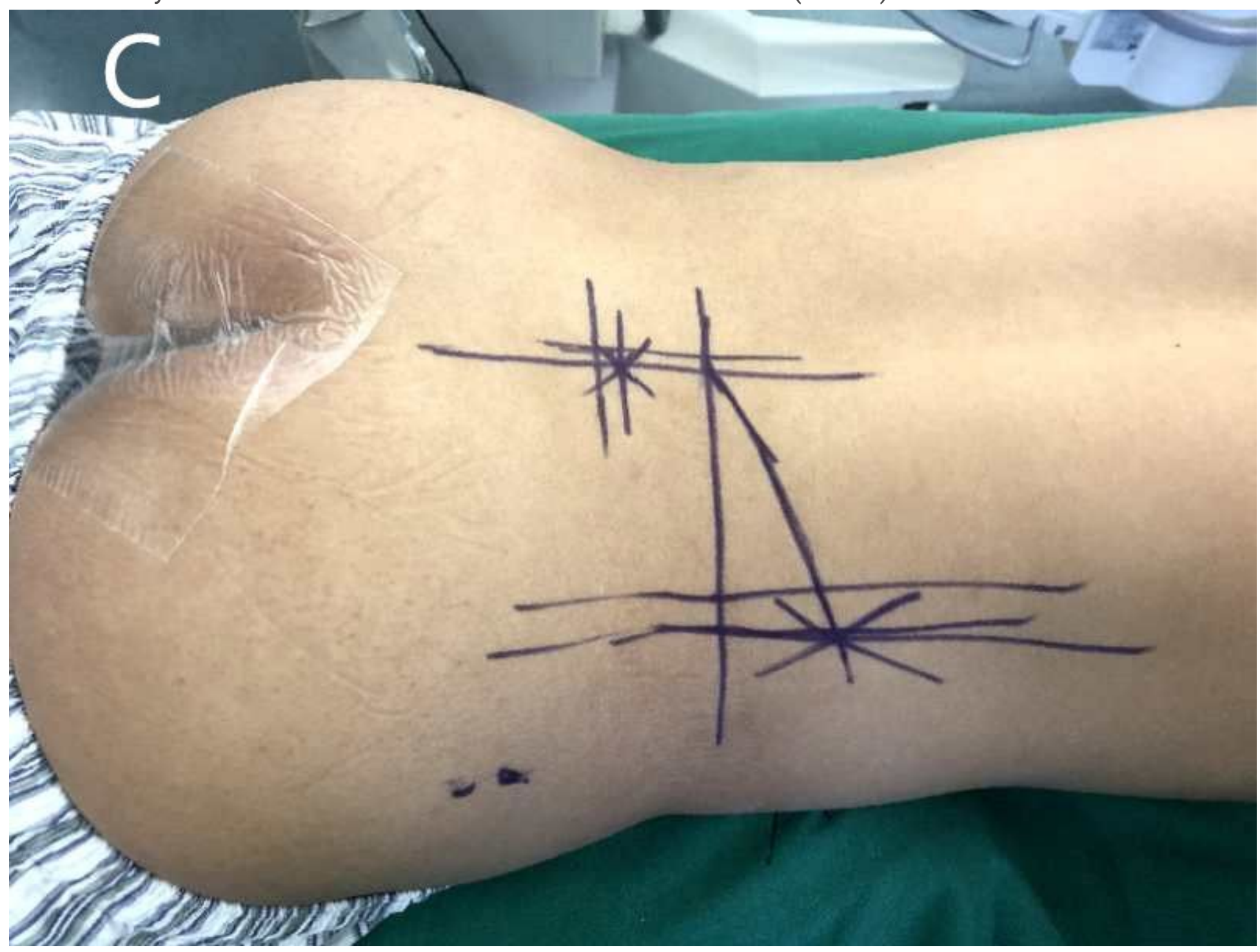

Figure 3

C-arm lateral image intensifier showing the foramina were enlarged with a saw 


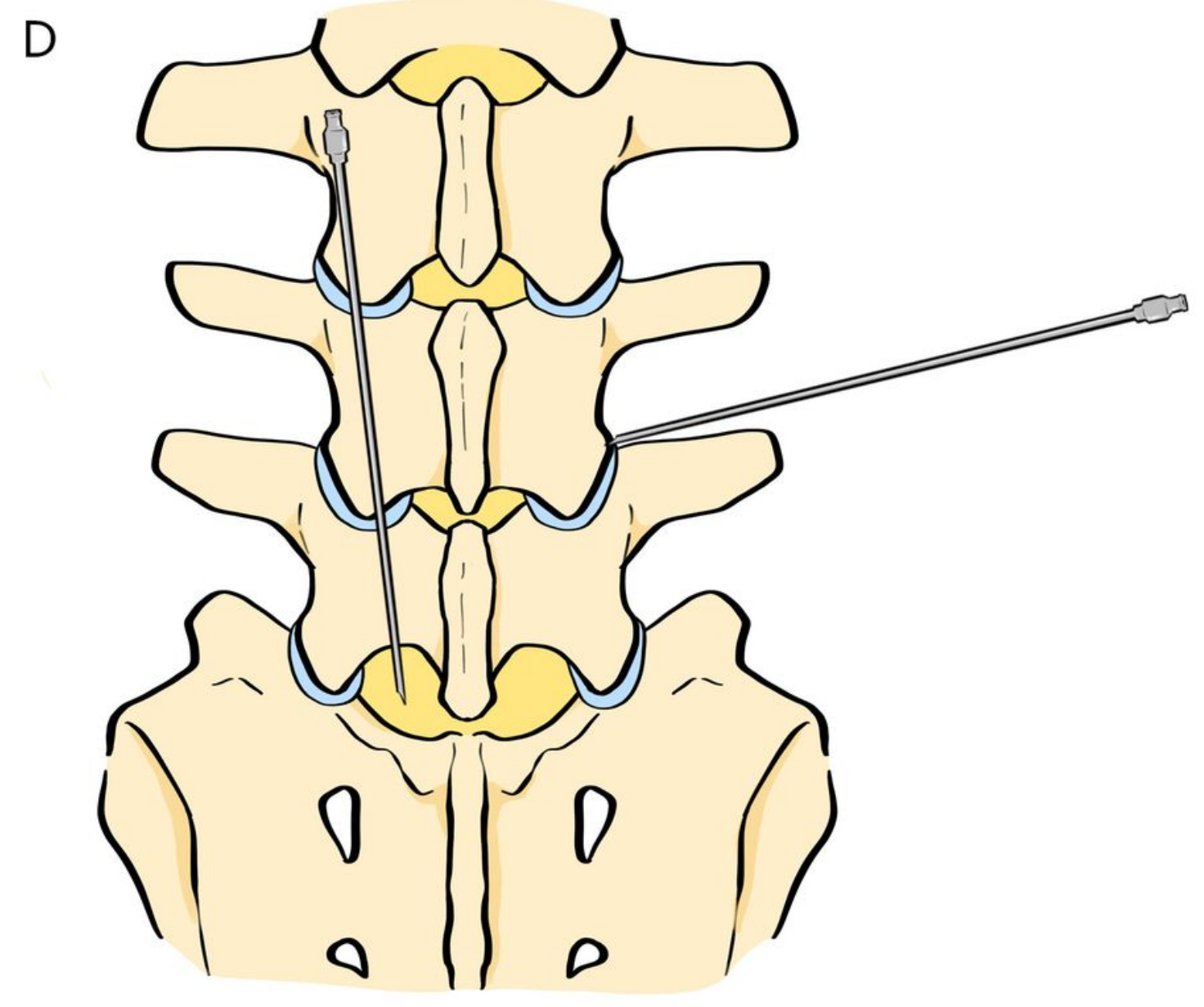

Figure 4

Long 18-gauge spinal needle was inserted from the entry point toward disk under fluoroscopic guidance 


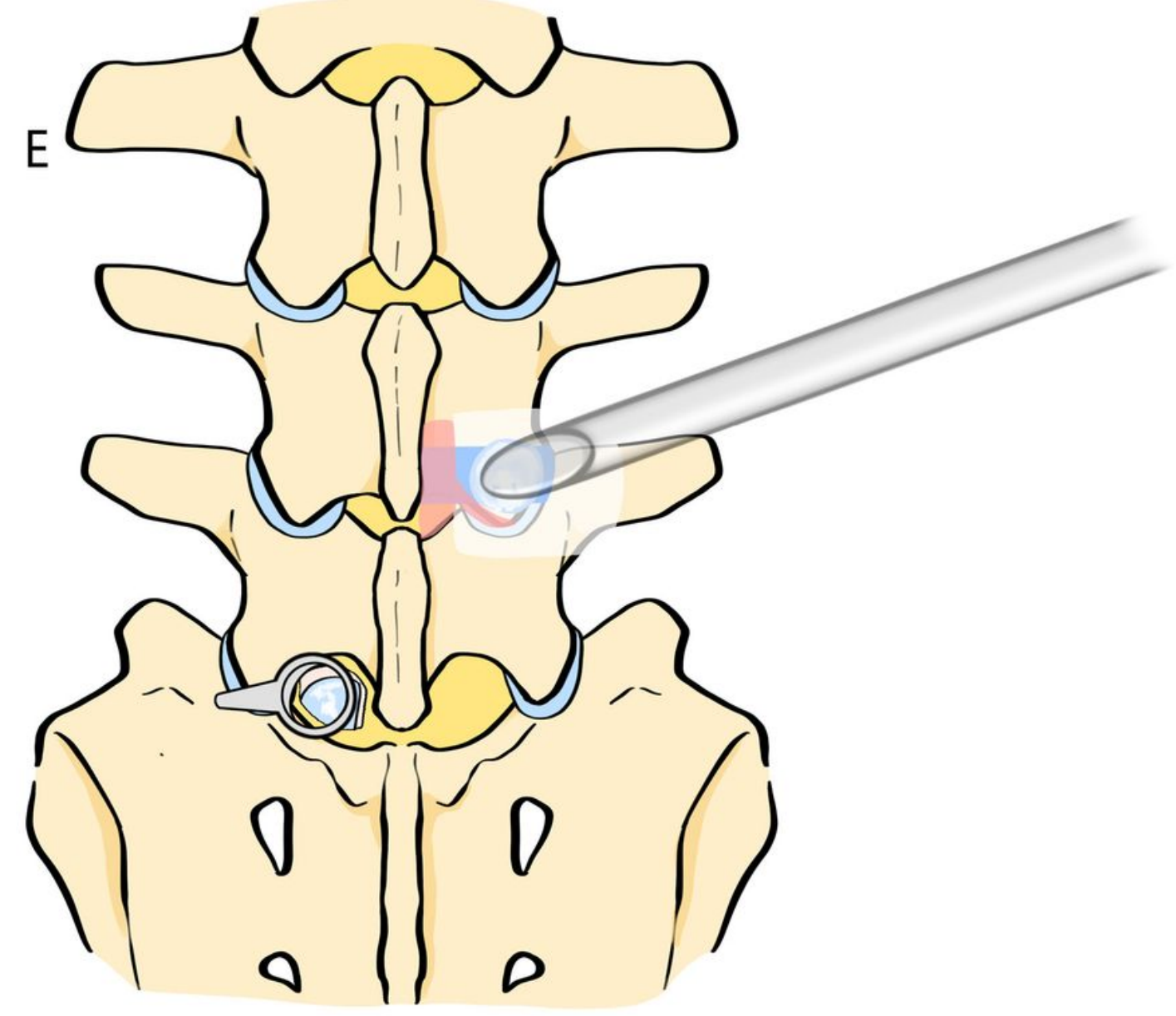

Figure 5

Diagrammatic drawing intraoperatively showing the foramina were enlarged alternately with a saw 


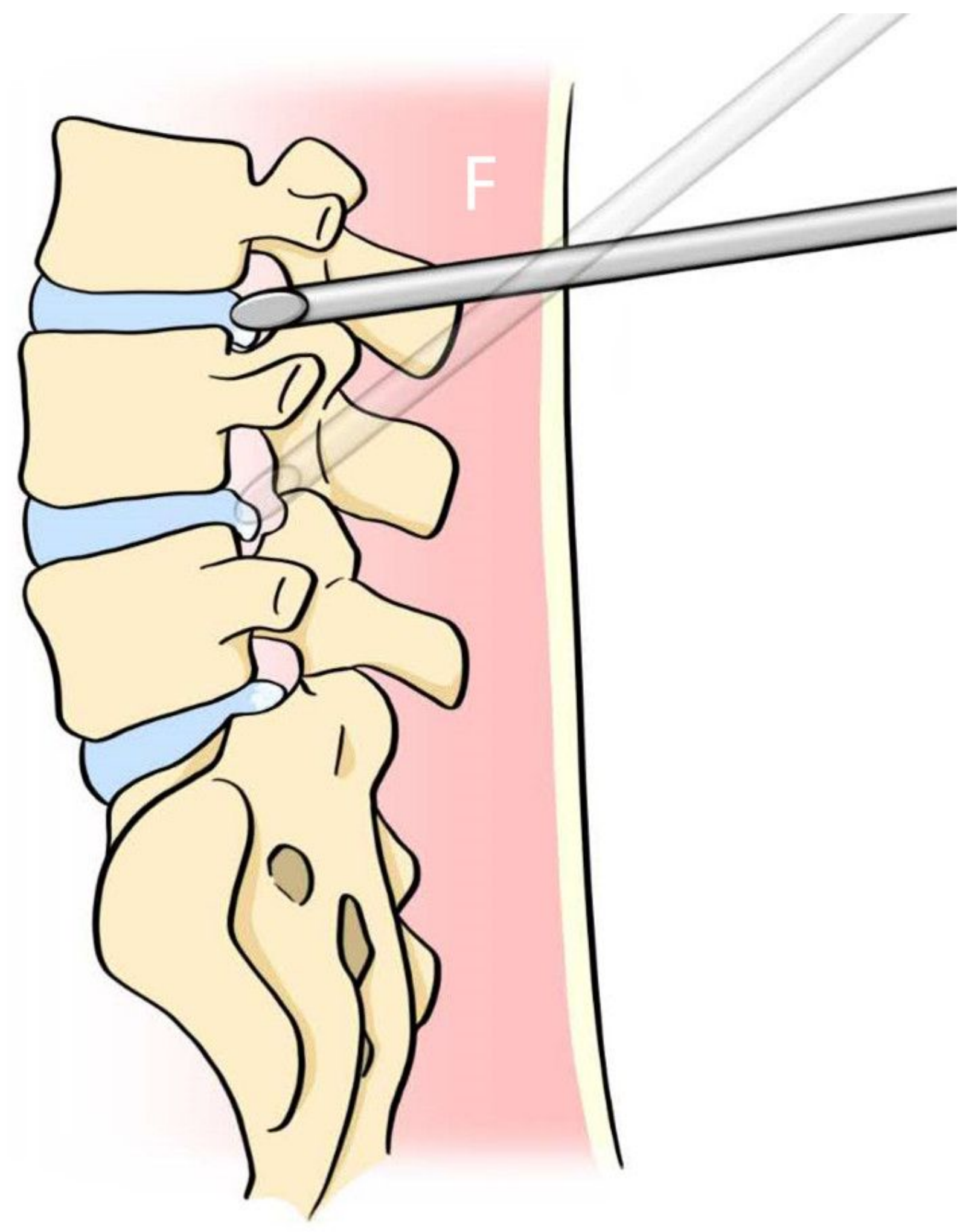

Figure 6

Diagrammatic drawing intraoperatively showing working cannula 


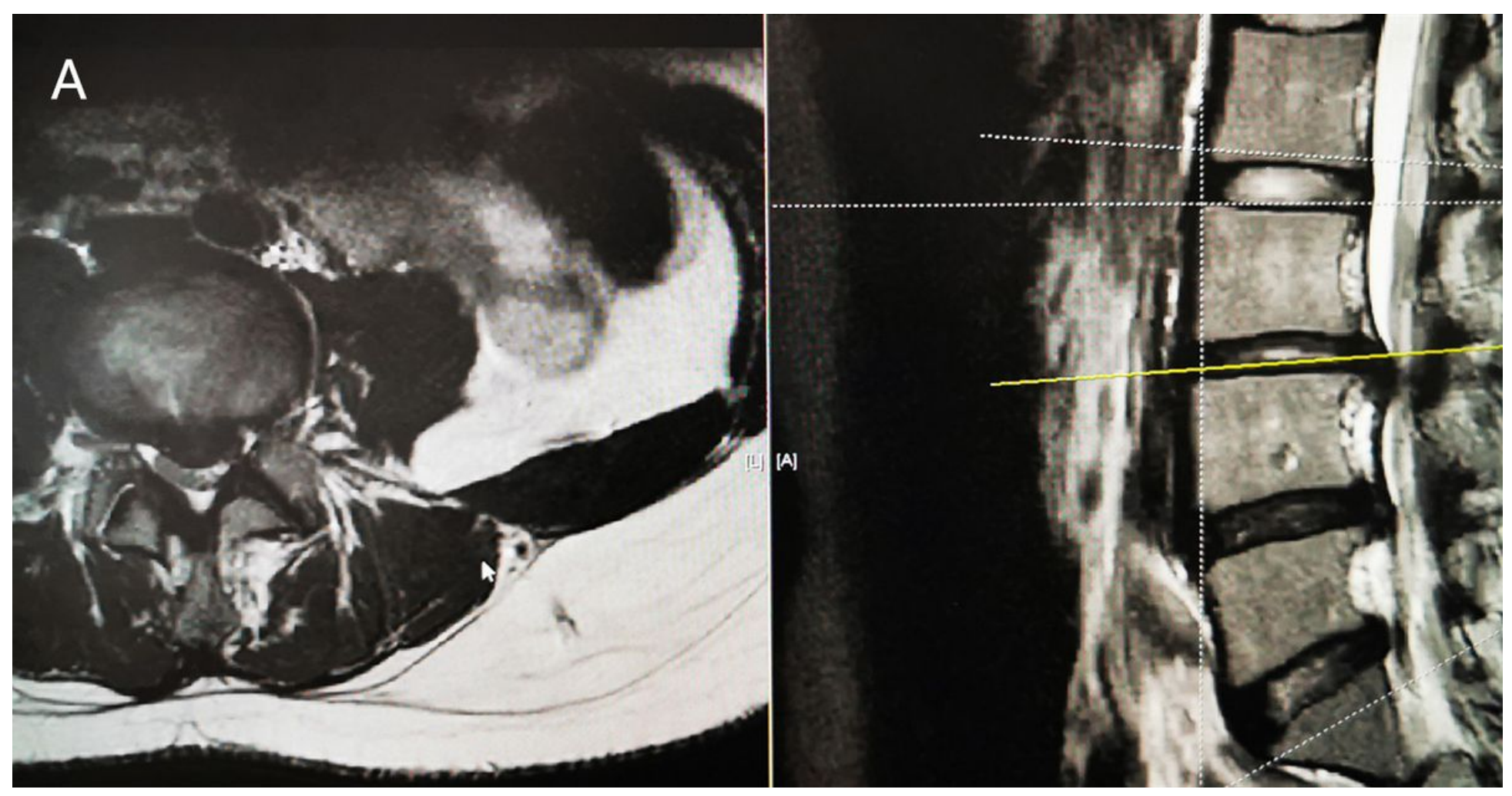

Figure 7

MRI of a 19-year-old man with lever L4/5 lumbar disc herniation (left).

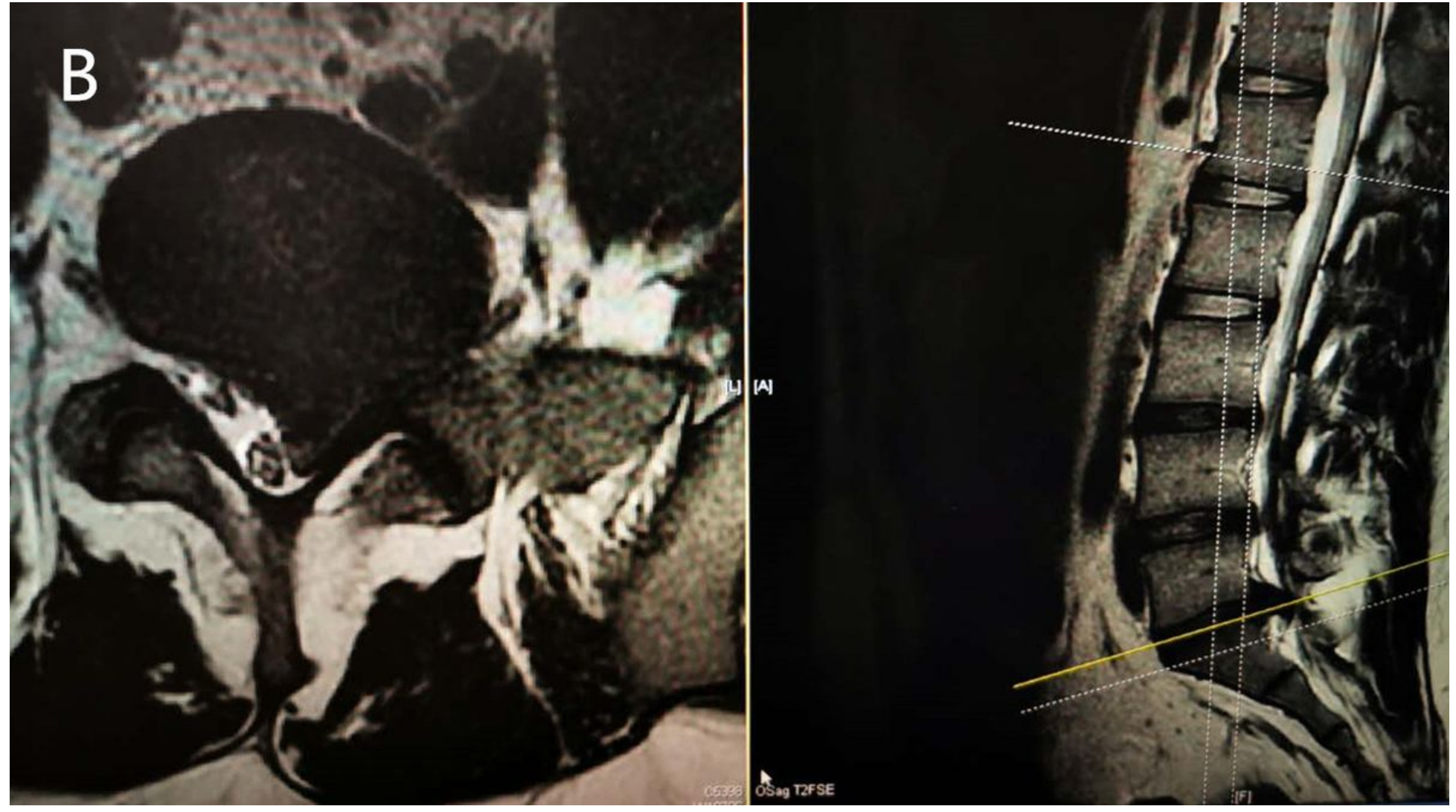

Figure 8 
MRI of a 19-year-old man with lever L5/S1 lumbar disc herniation (right).

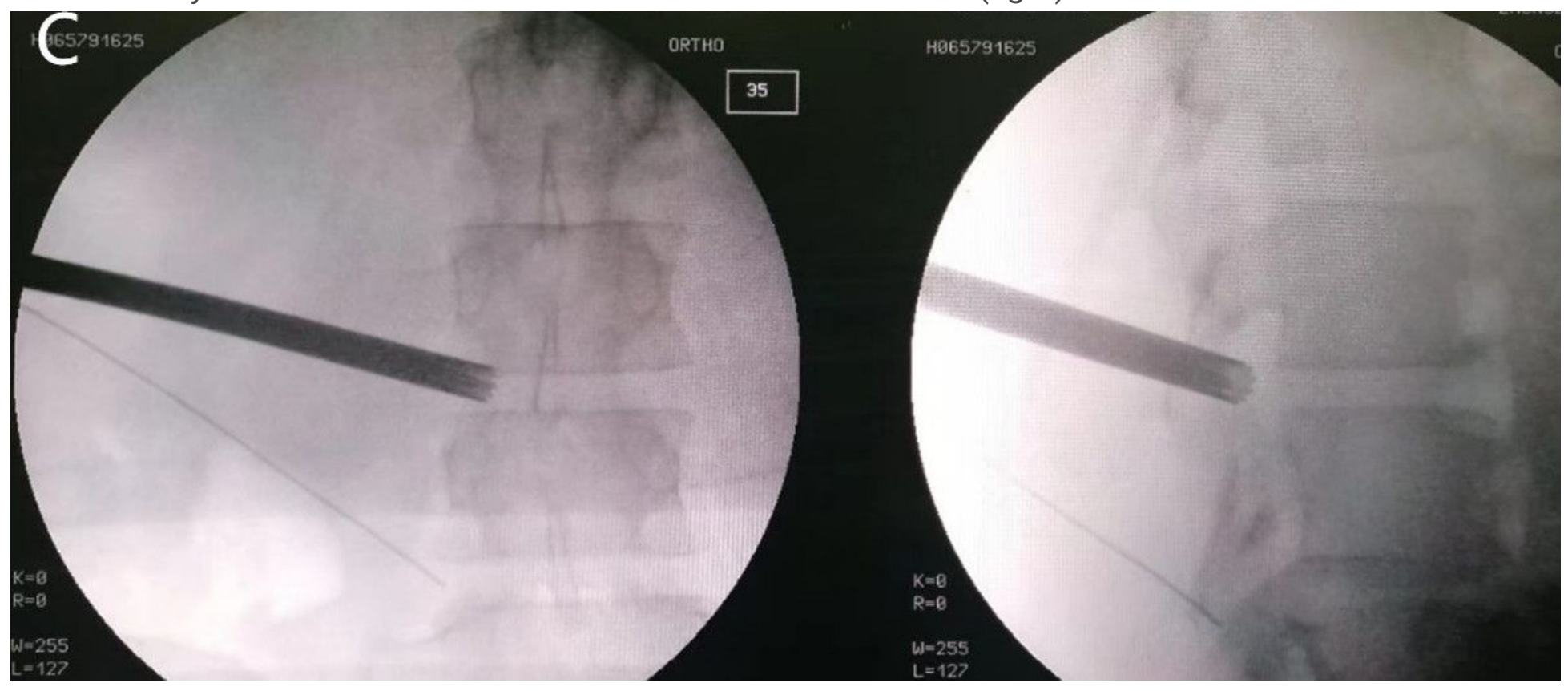

Figure 9

preoperative skin-marking of two puncture point for combined transforaminal and interlaminar FELD 


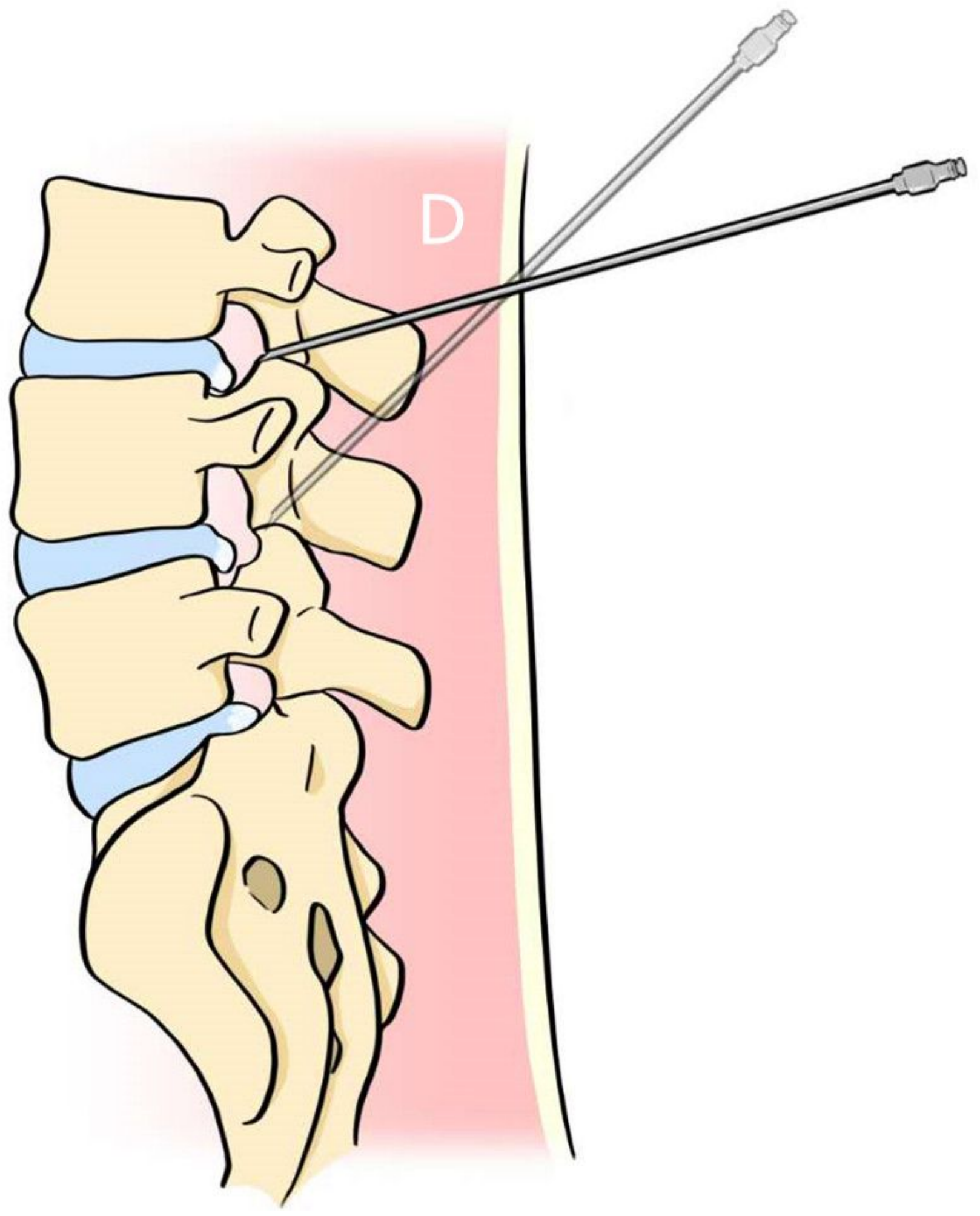

Figure 10

Long 18-gauge spinal needle was inserted alternately from the entry point toward disk under fluoroscopic guidance 


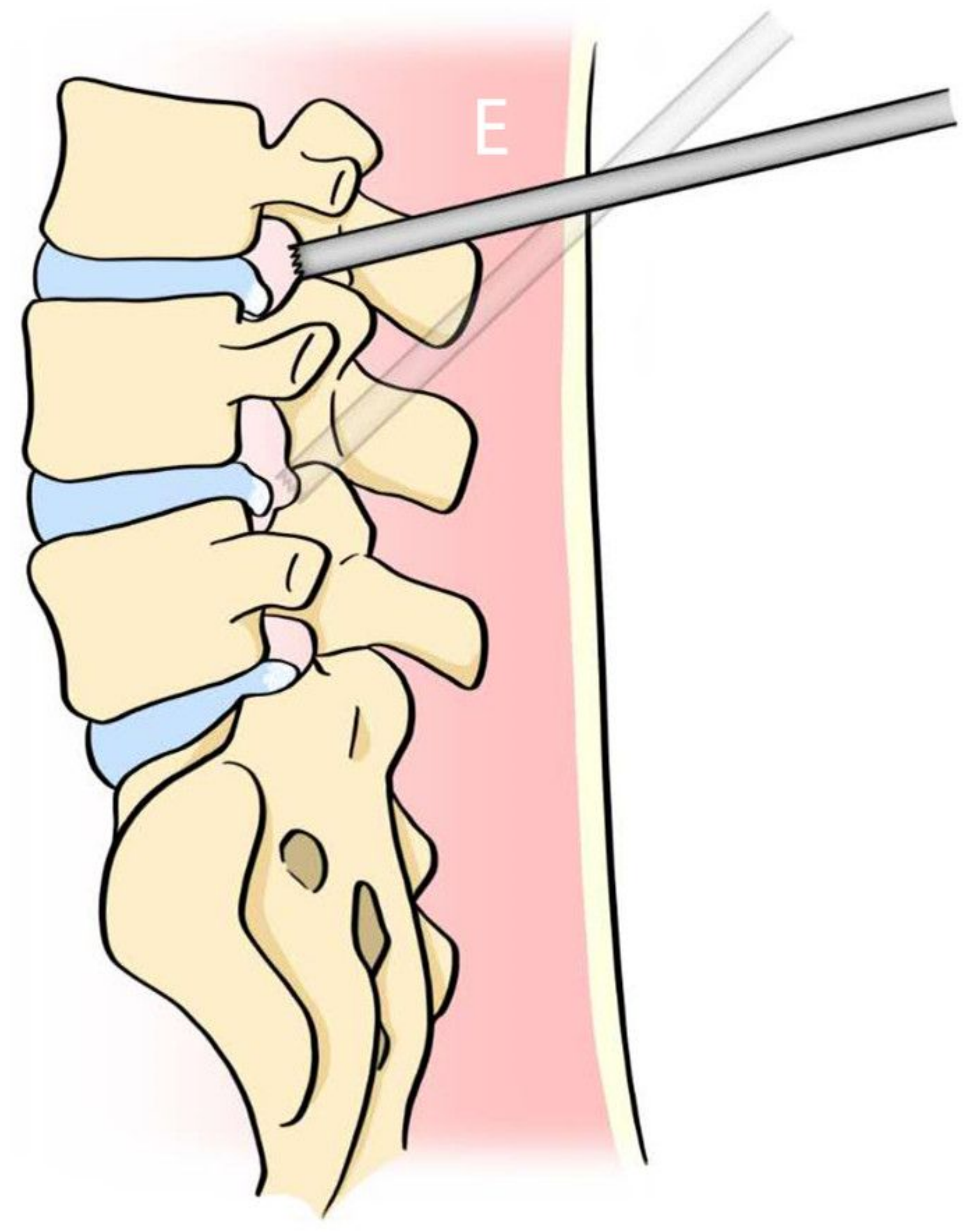

Figure 11

Diagrammatic drawing intraoperatively showing working cannula of combined transforaminal and interlaminar FELD 


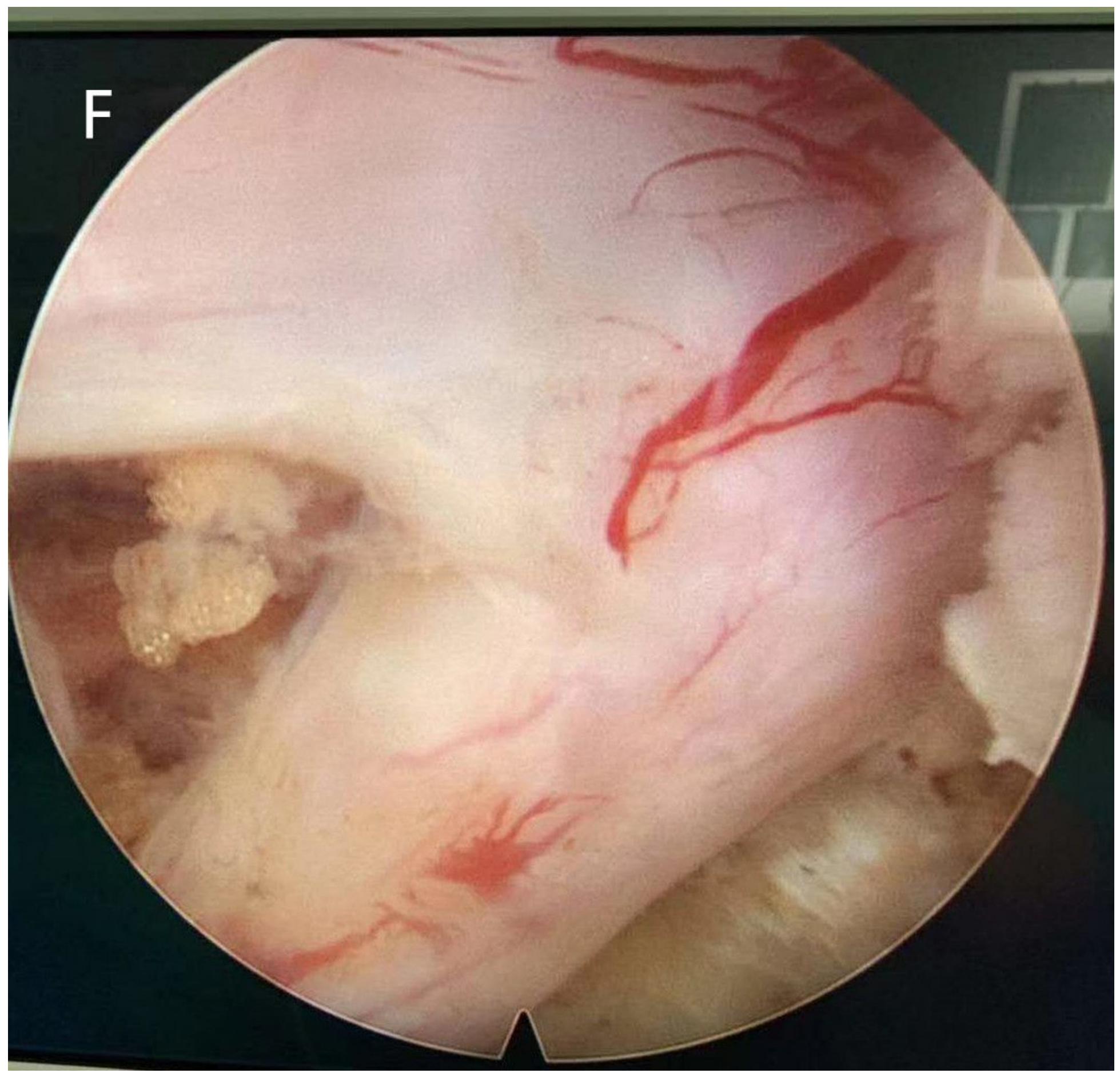

\section{Figure 12}

Intraoperative endoscopic view of nerve root was decompressed thoroughly.

\section{Supplementary Files}

This is a list of supplementary files associated with this preprint. Click to download.

- 6References.pdf 